\title{
Bowen's disease
}

\section{Kaoutar Laamari', Salim Gallouj', Fatima Zahra Mernissi ${ }^{1}$, Abdelhafid El Marfi ${ }^{2}$, Abdelmajid El Mrini²}

${ }^{1}$ Department of Dermatology, University Hospital Hassan II Fez, Morocco, ${ }^{2}$ Department of Traumatology Orthopedy B4, University Hospital Hassan II Fez, Morocco

Corresponding author: Dr. Kaoutar Laamari, E-mail: kaoutarlaamari1@gmail.com

A 32-year-old patient, with no significant pathological history, who consulted in dermatology for a nail lesion involving the left major, non-painless, nonpruritic, gradually increasing in size since 1 year. On the dermatological examination, it was noted the presence of a plate making $1,5 \mathrm{~cm}$ long axis, well limited, with verrucous surface sitting at the level of the distal edge of the left major with extension under one nail (Fig. 1). A cutaneous biopsy was performed on the tumor whose histology favored carcinoma in situ. Surgical treatment was indicated. Bowen's disease is a carcinoma that rarely affects the nail system. It usually presents as an erythematous plaque or hyperkeratotic verrucous lesions. Nodules, ulcerations and bleeding are also described. Treatment remains surgical.

\section{Consent}

The examination of the patient was conducted according to the Declaration of Helsinki principles.

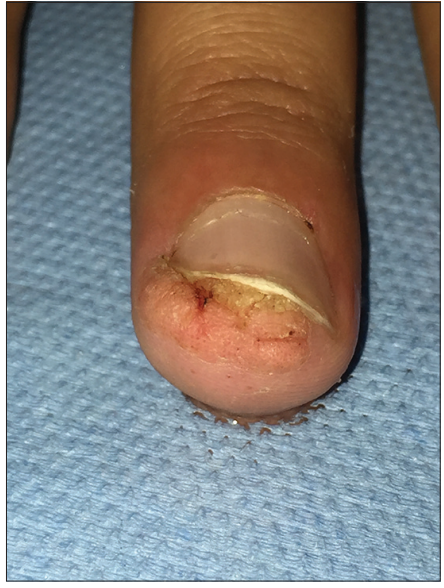

Figure 1: A plate making 1,5cm long axis, well limited, with verrucous surface sitting at the level of the distal edge of the left major with extension under one nail.

Copyright by Kaoutar Laamari, et al. This is an open-access article distributed under the terms of the Creative Commons Attribution License, which permits unrestricted use, distribution, and reproduction in any medium, provided the original author and source are credited.

Source of Support: Nil, Conflict of Interest: None declared. 